\title{
Multidrug-resistant Klebsiella pneumoniae: a retrospective study in Manaus, Brazil
}

\author{
Rafael Nakamura-Silva ${ }^{1} \cdot$ Louise Cerdeira $^{2,3} \cdot$ Mariana Oliveira-Silva ${ }^{1} \cdot$ Karen Regina Carim da Costa ${ }^{4}$. Elder Sano ${ }^{5,6}$. \\ Bruna Fuga $^{5,6,7} \cdot$ Quézia Moura ${ }^{8} \cdot$ Fernanda Esposito $^{6,7} \cdot$ Nilton Lincopan $^{5,6,7} \cdot$ Kelly Wyres $^{2} \cdot$ André Pitondo-Silva $^{1,9}$
}

Received: 28 October 2021 / Revised: 6 February 2022 / Accepted: 18 February 2022 / Published online: 4 March 2022

(c) The Author(s), under exclusive licence to Springer-Verlag GmbH Germany, part of Springer Nature 2022

\begin{abstract}
Klebsiella pneumoniae is an opportunistic pathogen that can cause several infections, mainly in hospitalised or immunocompromised individuals. The spread of K. pneumoniae emerging virulent and multidrug-resistant clones is a worldwide concern and its identification is crucial to control these strains especially in hospitals. This article reports data related to multi-resistant K. pneumoniae strains, isolated from inpatients in the city of Manaus, Brazil, harbouring virulence and antimicrobialresistance genes, including high-risk international clones belonging to clonal group (CG) 258. Twenty-one strains isolated from different patients admitted to four hospitals in the city of Manaus, located in the state of Amazonas, Northern Brazil (Amazon Rainforest region) were evaluated. The majority of strains $(61.9 \% n=13)$ were classified as multidrug-resistant (MDR), and five strains (23.8\%) as extensively drug-resistant (XDR). Several virulence and antimicrobial-resistance genes were found among the strains and eight strains (38.1\%) presented the hyper-mucoviscous phenotype. MLST analysis demonstrated a great diversity of STs among the strains, totaling 12 different STs (ST11, ST23, ST198, ST277, ST307, ST340, ST378, ST462, ST502, ST3991, ST3993 and ST5209). Three of these (ST11, ST23 and ST340) belong to CG258.
\end{abstract}

Keywords Klebsiella pneumoniae · Resistome · Virulome · Hypermucoviscous · WGS · CG258

\section{Introduction}

Klebsiella pneumoniae is a member of the Enterobacteriaceae family and an opportunistic pathogen that could cause several infections (pneumonia, urinary tract infections,

Communicated by Erko Stackebrandt.

Rafael Nakamura-Silva and Louise Cerdeira contributed equally to this work.

André Pitondo-Silva

andre@pitondo.com.br

1 Postgraduate Program in Environmental Technology, Universidade de Ribeirão Preto, UNAERP, Bloco J, Laboratório 1. Av. Costábile Romano, 2201 Ribeirânia, Ribeirão Preto, São Paulo 14096-900, Brazil

2 Department of Infectious Diseases, Central Clinical School, Monash University, Melbourne, Victoria, Australia

3 Department of Vector Biology, Liverpool School of Tropical Medicine, Liverpool, UK

4 School of Pharmaceutical Sciences, Universidade Federal do Amazonas, Manaus, Amazonas, Brazil bacteremia, among others), mainly in hospitalised or immunocompromised individuals (Bengoeche et al. 2019).

The appearance and spread of hyper-virulent strains have increased the number of people susceptible to infections; also, strains of $K$. pneumoniae have become increasingly resistant to antibiotics, making antibiotic therapy more challenging. Several new antimicrobial-resistance genes were discovered in $K$. pneumoniae before spreading to other pathogens; $b l a_{\mathrm{KPC}}, b l a_{\mathrm{OXA}-48-\text { like }}$ and $b l a_{\mathrm{NDM}-1}$ are examples

5 Institute of Biomedical Sciences, Universidade de São Paulo, São Paulo, São Paulo, Brazil

6 One Health Brazilian Resistance Project (OneBR), São Paulo, São Paulo, Brazil

7 Department of Clinical Analysis, School of Pharmaceutical Sciences, University of São Paulo, São Paulo, São Paulo, Brazil

8 Federal Institute of Education, Science and Technology of Espírito Santo, Vila Velha, Espírito Santo, Brazil

9 Postgraduate Program in Dentistry, Universidade de Ribeirão Preto, Ribeirão Preto, São Paulo, Brazil 
(Wyres et al. 2018). Molecular epidemiology analyses allow us to determine the global spread of high-risk clones, thus, providing the necessary data to develop strategies to limit the spread of clinically dangerous strains (Mathers et al. 2015).

The purpose of this study was to determine the pathogenic potential and the antimicrobial-resistance profiles of $K$. pneumoniae strains isolated from different patients admitted to four hospitals in the city of Manaus, located in the state of Amazonas, Northern Brazil. Besides, to analyse the genetic diversity and epidemiological relationship of the strains, Manaus is a Brazilian municipality, Amazonas capital, and the leading financial centre in the North of the country. It is the most populous city in the entire Brazilian Amazon, located in the centre of the world's largest tropical forest (IBGE 2019).

Recently, the city suffered a collapse in the public health system due to the COVID-19 pandemic (Ferrante et al. 2020). Therefore, studies even before the pandemic period aiming to elucidate and prevent the spread of hyper-virulent and multidrug-resistant bacteria in hospitals in this region are critical (Ribas et al. 2020).

\section{Materials and methods}

\section{Bacterial strains}

In this study, $21 \mathrm{~K}$. pneumoniae strains isolated from different patients admitted to four hospitals in the city of Manaus, located in the state of Amazonas, Northern Brazil, were evaluated. The strains were randomly selected from November 2014 to May 2016, from different sources, including urine, blood, tracheal secretion, wound secretion, catheter tip, rectal swab (Fig. 1). Bacterial species were initially identified in the hospitals where they were isolated by the automated system VITEK 2 (Biomérieux). Subsequently, to guarantee the correct identification of the species, all strains were molecularly identified by $16 \mathrm{~S}$ rRNA sequencing using the primers fD1 (5'-AGAGTTTGATCCTGGCTCAG-3') and rP2 (5'-ACGGCTACCTTGTTACGACTT-3'), according to Weisburg et al. (1991).

\section{Hypermucoviscosity test}

For the detection of hyper-mucoviscous phenotype (HMV), all strains were inoculated on Mueller-Hinton agar (Oxoid),
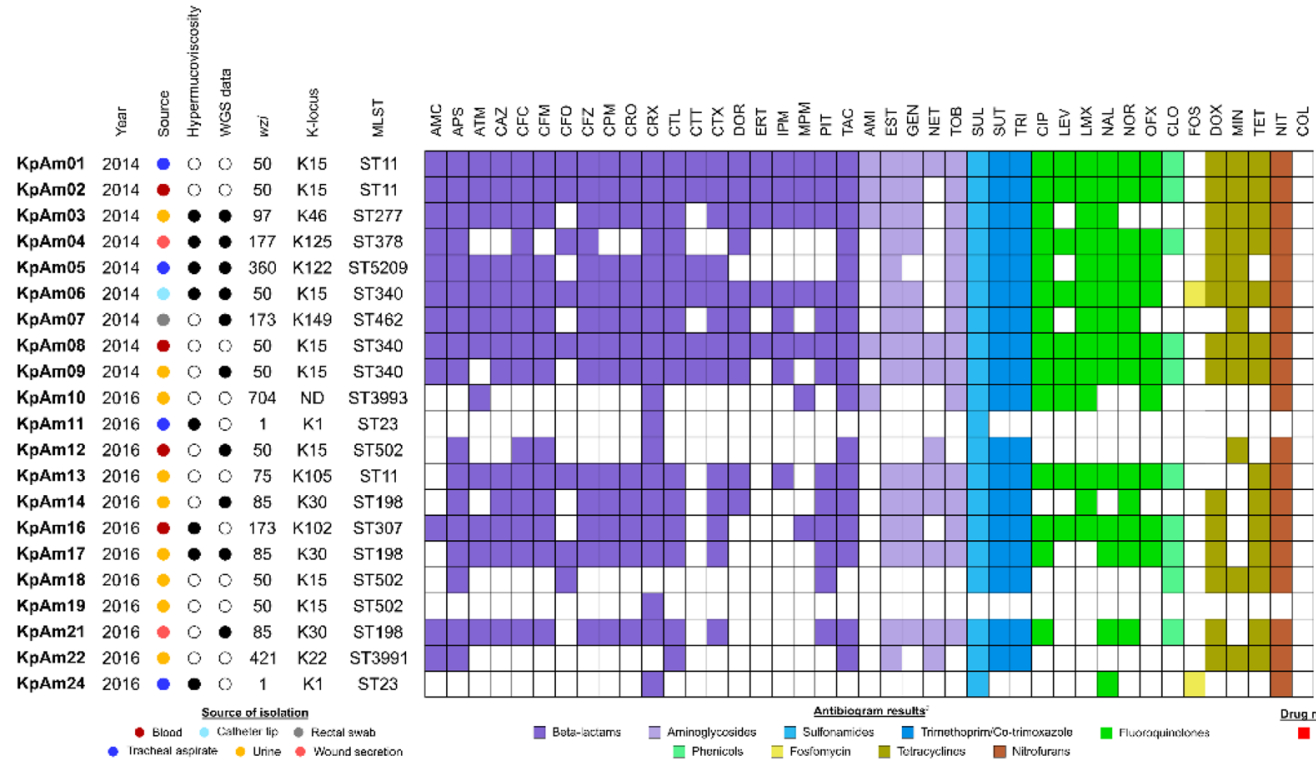

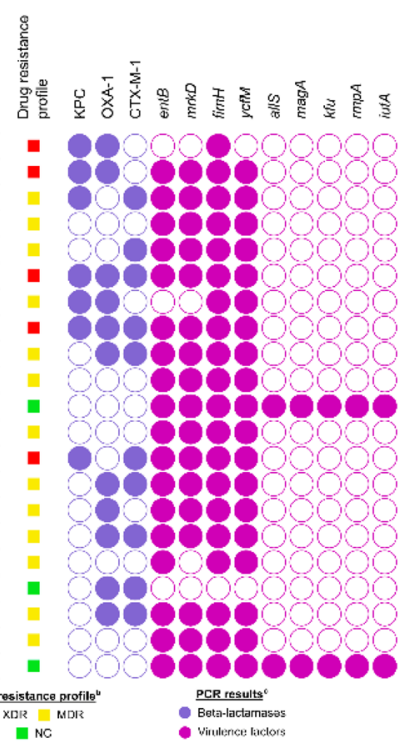

Fig. 1 Phenotypic and molecular characterisation of 21 Klebsiella pneumoniae isolates from clinical sources in Manaus, Brazil. Antimicrobials tested: amoxicillin-clavulanate (AMC), amikacin (AMI), ampicillin-sulbactam (APS), aztreonam (ATM), ceftazidime (CAZ), cefaclor (CFC), cefixime (CFM), cefoxitin (CFO), cefazolin (CFZ), ciprofloxacin (CIP), chloramphenicol (CLO), colistin (COL), cefepime (CPM), ceftriaxone (CRO), cefuroxime (CRX), ceftaroline (CTL), cefotetan (CTT), cefotaxime (CTX), doripenem (DOR), doxycycline (DOX), ertapenem (ERT), streptomycin (EST), fosfomicin (FOS), gentamicin (GEN), imipenem (IPM), levofloxacin (LEV), lomefloxacin (LMX), minocycline (MIN), meropenem (MPM), nalidixic acid (NAL), netilmicin (NET), nitrofurantoin (NIT), norfloxacin (NOR), ofloxacin (OFX), piperacillin-tazobactam (PIT), sulphonamide (SUL), trimethoprim-sulfamethoxazole (SUT), ticarcillin-clavulanate (TAC), tetracycline (TET), tobramycin (TOB), trimethoprim (TRI). ${ }^{a}$ Colored square: non-susceptible strains; blank squares: susceptible strains. No color was associated to polymyxins, since all strains analysed was susceptible to colistin. ${ }^{\mathrm{b}} \mathrm{XDR}$ extensively drugresistant, $M D R$ multidrug-resistant, $N C$ not classified. ${ }^{c}$ Filled circles: detected; unfilled circles: not detected 
for approximately $18 \mathrm{~h}$ at $37^{\circ} \mathrm{C}$. Following bacterial growth, using the bacteriological loop, an isolated colony was touched and raised vertically. The formation of a dense string $\geq 5 \mathrm{~mm}$ was considered a positive HMV phenotype, according to Wiskur et al. (2008).

\section{Antimicrobial susceptibility test}

Antimicrobial susceptibility tests were performed by disc diffusion with 40 different antibiotics on Mueller-Hinton agar (Oxoid), according to Clinical Laboratory Standards Institute (CLSI 2020) recommendations for Enterobacterales. Susceptibility to colistin was determined by microdilution, according to CLSI 2020. The antibiotics tested are described in Fig. 1. Escherichia coli ATCC 25922 and Pseudomonas aeruginosa ATCC 27853 were used as controls in this experiment.

Each strain was considered susceptible or non-susceptible (either intermediate or resistant) to each antibiotic tested. Based on the susceptibility profile, the strains were classified as multidrug-resistant (MDR), extensively drug-resistant (XDR) or pandrug-resistant (PDR), according to Magiorakos et al. (2012).

\section{Detection of virulence and $\beta$-lactamase encoding genes}

All strains were subjected to Polymerase Chain Reaction (PCR) assays to detect 10 virulence genes ( $r m p A, k f u$, alls, fimH, mrkD, ycfM, ent $B$, iutA, $\mathrm{K} 2$, magA) and $15 \beta$ - lactamase encoding genes, including carbapenemases $\left(b l a_{\mathrm{GES}}\right.$, $b l a_{\mathrm{IMP}}, b l a_{\mathrm{KPC}}, b l a_{\mathrm{NDM}}, b l a_{\mathrm{OXA}-1-\mathrm{like}}, b l a_{\mathrm{OXA}-48-\mathrm{like}}, b l a_{\mathrm{VIM}}$, $b l a_{\mathrm{SPM}}$ and $\left.b l a_{\mathrm{GIM}}\right)$, extended-spectrum $\beta$-lactamasesESBLs $\left(b l a_{\text {СTX-M-Gp1 }}, b l a_{\text {CTX-M-Gp2 }}, b l a_{\text {СTX-М-Gр9 }}, b l a_{\text {CMY-2}}\right.$, $b l a_{\mathrm{VEB}}$ e $\left.b l a_{\mathrm{BEL}}\right)$ and also colistin-resistance gene ( $m c r$ 1). The protocols used to investigate the virulence and $\beta$-lactamase encoding genes were performed as described by Nakamura-Silva et al. (2021b).

To confirm the detected genes' identity, an amplicon from each gene was randomly selected for DNA sequencing (ABI 3500xL Genetic Analyzer; Applied Biosystems, Foster City, CA). The obtained sequences were compared with those available in GenBank using the BLAST algorithm (http:// blast.ncbi.nlm.nih.gov/Blast.cgi).

\section{Multilocus sequence typing (MLST)}

To determine sequence types and evaluate the clonal relationships, all strains were submitted to the MLST technique using the primers and PCR conditions for protocol 2 in the MLST database for K. pneumoniae (http://www.pasteur.fr/ recherche/genopole/PF8/mlst/Kpneumoniae.html).

\section{Whole-genome sequencing (WGS) and bioinformatic analysis}

Aiming a more detailed analysis to elucidate the resistome, viruloma and plasmidoma, ten strains (KpAm03, KpAm04, KpAm05, KpAm06, KpAm07, KpAm09, KpAm12, KpAm14, KpAm17 and KpAm21) were subjected to wholegenome sequencing (WGS), which were selected based on the antimicrobial-resistance classification, presence of hyper-mucoviscosity and virulence genes.

Total genomic DNA was extracted using a PureLinkTM Quick Gel Extraction Kit (Life Technologies, CA). Genomic DNA quality and quantity were assessed using a Qubit1 2.0 fluorometer (Life Technologies) and sequenced via $2 \times 150$ bp paired-end library on a NextSeq550 or MiSeq platform (Illumina Inc., San Diego, CA), using 250-bp paired-end (PE) reads (Table supplementary 1). Reads with a PHRED quality score below 20 were discarded, and adapters were trimmed using TrimGalore v0.6.5 (https://github.com/Felix Krueger/TrimGalore). The reads were de novo assembled using Unicycler v0.4.0 (Wick et al. 2017) and annotated using the Prokaryotic Genome Annotation Pipeline v.3.2 (PGAP) NCBI.

Antimicrobial-resistance (AMR) genes were identified using ABRicate v1.0.1 (https://github.com/tseemann/abric ate) with ResFinder v4.1 database (Bortolaia et al. 2020) and a database for heavy metals and biocides-resistance genes. ABRicate was also used with PlasmidFinder v2.1 database (Carattoli et al. 2014) to detect plasmid replicons among the assemblies contigs. MLST, virulence factors, capsule synthesis (K-locus), lipopolysaccharide (O-locus), and mutations on porins and quinolone resistance determining regions were identified using Kleborate v2.0.1 (Lam et al. 2021).

CSI Phylogeny (Kaas et al. 2014) was used to generate an approximately maximum-likelihood phylogenetic tree the assemblies, using K. pneumoniae strain HS11286 chromosome (RefSeq accession number NC_016845.1) as reference. The tree was rooted at midpoint and annotated with data from ABRicate and Kleborate, as well as metadata, using iTOL v6 (Letunic et al. 2021). iTOL was also used with a dummy tree to generate heatmaps with antibiogram and PCR results for all the 21 strains.

\section{Results and discussion}

Infections caused by $K$. pneumoniae with multiple antibiotic-resistance genes, associated with several virulence factors are increasingly reported in hospitalized patients in Brazil and in different parts of the world. These infections have been a global concern, as the therapeutic limitations associated with the pathogenicity of many strains with MDR and XDR phenotypes are related with a large number of 
morbidity and mortality (Gonçalves et al., 2017; Campos et al., 2018; Lam et al., 2021; Nakamura-Silva et al. 2021b). Therefore, this study aimed to evaluate $21 \mathrm{~K}$. pneumoniae strains isolated from different patients admitted to four hospitals in Manaus, located in the state of Amazonas, northern Brazil.

All strains were subjected to antimicrobial susceptibility test, with a total of forty-one antibiotics of different classes (Fig. 1), most strains were non-susceptible to the class folate pathway antagonists, sulphonamide (SUL) $(95.2 \% n=20)$; trimethoprim-sulfamethoxazole (SUT) $(85.7 \%)$ and trimethoprim (TRI) (85.7\%); as well nitrofurantoin (NIT) and cefuroxime (CRX) (both 90.5\%); a high rate of non-susceptibility was also demonstrated for $\beta$-lactam combination agents, ticarcillin-clavulanate (TAC) and ampicillin-sulbactam (APS) (both 80.9\%). Non-susceptibility rates above 70\% were also observed for the antibiotics: streptomycin (EST), tobramycin (TOB), cefaclor (CFC) and ceftaroline (CTL). The results of non-susceptibility of each antibiotic are shown in Fig. 1. Similar results were described by Pereira et al. (2019) who evaluated E. coli (362 strains) and Klebsiella spp. (73 strains) isolated from urinary tract infections in southern Brazil, which demonstrated high percentage of resistance in $91.3 \%(n=21 / 23)$ antimicrobials analysed: ampicillin (AMP), ampicillin-sulbactam (APS), piperacillin-tazobactam (PIT), amoxicillin-clavulanate (AMC), cefazolin (CFZ), cefotaxime (CTX), ceftazidime (CAZ), ceftriaxone (CRO), cefuroxime (CRX), cefepime (CPM), cefoxitin (CFO), aztreonam (ATM), ertapenem (ERT), meropenem (MPM), imipenem (IPM), amikacin (AMI), tobramycin (TOB), nitrofurantoin (NIT), sulfamethoxazole (SUT), tetracycline (TET), ciprofloxacin (CIP), levofloxacin (LEV). The exceptions are APS and IPM.

The majority of strains $(61.9 \% n=13)$ were classified as MDR and five strains (23.8\%) were classified as XDR (Fig. 1). These high rates of MDR and XDR phenotypes have been reported in other studies carried out in Brazil. Ferreira et al. (2019) investigated the resistance profile of $25 \mathrm{~K}$. pneumoniae from the Tocantins, northern Brazil, finding a high incidence of MDR among them (84\%). Gonçalves et al. (2017) studied $26 \mathrm{~K}$. pneumoniae strains from patients admitted in a tertiary hospital in Londrina, southern Brazil, being 53.8\% classified as MDR and 26.9\% as XDR and $11.5 \%$ PDR.

PCR analyses detected in eleven strains (52.4\%) presence of the $b l a_{\text {OXA-1-like }}$ genes, ten with $b l a_{\text {CTX-M-Gp1 }}$ genes (47.6\%) and seven strains harboring bla $a_{\mathrm{KPC}}(33.3 \%)$. Accession numbers MT330306, MT330308 and MT330310. The XDR KpAm06 and KpAm08 encoded all the three of these $\beta$-lactamases genes, another ten strains (KpAm01, KpAm02, KpAm03, KpAm07, KpAm09, KpAm13, KpAm14, $\mathrm{KpAm17,} \mathrm{KpAm19} \mathrm{and} \mathrm{KpAm21)} \mathrm{harboured} \mathrm{two} \mathrm{of} \mathrm{these}$ three $\beta$-lactamases. The same genes have been reported in $K$. pneumoniae from similar studies in southern, southeastern and northern Brazil (Gonçalves et al. 2017; Azevedo et al. 2019; Ferreira et al. 2019).

Among the virulence genes, the $f i m H$ gene was detected in majority strains $(95.2 \% n=20)$, the second-most prevalent gene was $y c f M(90.5 \% n=19)$, followed by entB $(85.7 \%$ $n=18)$ and $m r k D(80.9 \% n=17)$ (Fig. 1). Accession numbers of all detected virulence genes were MT330312, MT330313, МT330314, МT330316, MT330317, MT330319, MT330320, MT330322 and MT330324. Remya et al. (2019) studied virulence factors in $370 \mathrm{~K}$. pneumoniae clinical strains and showed that entB was present in $90 \%$ of the strains, fimH $89.1 \%, y b t S 44.3 \%$, kfu $27.8 \%$, rmpA $5.1 \%$, K2 2\%, alls $1 \%$ and magA $0.2 \%$. The large detection of entB and fimH; reasonable $y b t S$ and; low rmpA and $m a g A$ are similar to our results. Fimbrial adhesins ( fimH, $m r k \mathrm{D}$ ), siderophores (ent $\mathrm{B}$, mainly) and lipopolysaccharides $(y c f M)$ were the most common genes in this study and similar results were found by Candan et al. (2015), with 74\% $y c f M$ and $65 \%$ of $m r k D, f i m H$ and $e n t B$, which studied $15 \mathrm{~K}$. pneumoniae clinical strains isolated from different sources. The high prevalence of $f i m H, m r k D$ and entB genes found in this study was already expected, other studies have shown a high correlation of these genes in K. pneumoniae strains (Azevedo et al. 2019; Ferreira et al. 2019; Kus et al. 2017). The strain KpAm11 presented nine of the virulence genes studied, only K2 was not found in this strain (Fig. 1). Eight strains (38.1\%) presented the HMV phenotype (Fig. 1), the rmpA2 (mucoid phenotype A2 regulator) and $m a g A$ genes (mucoviscosity-associated A gene) were associated with HMV phenotype, however, subsequent studies showed that the magA is associated to specific capsular serotype $\mathrm{K} 1$ (wzy K1) (Catalán-Nájera et al. 2017). Among the eight strains positive for this phenotypic in our study, only KpAm11 and KpAm24 presented magA and $r m p A$ genes. Similar results were found by Lee et al. (2010) which found three (8\%) among 35 strains that presented the phenotype without the presence of at least one of the three genes, $m a g A, r m p A$ and rmpA2. Other studies also showed equivalents results, $10.3 \%$ ( $n=6 / 58)$ HMV K. pneumoniae from Taiwan did not have the magA and rmpA genes (Yu et al. 2006) and among the results of Mohammed et al. (2018), one out of six clinical strains demonstrated the characteristic in question. Moreover, our findings corroborate the results recently described by our research group (Nakamura-Silva et al. 2021a) and also by Garza-Ramos (2015) who did not find these genes in $K$. variicola HMV strains. These results indicate that other genes may be involved in this phenotype in different species of Klebsiella which need further investigation. Walker et al. (2020) suggest that the HMV phenotype is probably due to factors not exclusive to the capsule. These authors suggest that it is necessary to investigate and clarify the link between the HMV phenotype and the capsule. 
MLST analysis demonstrated a great diversity of STs among the strains, totaling 12 different STs (ST11, ST23, ST198, ST277, ST307, ST340, ST378, ST462, ST502, ST3991, ST3993 and ST5209). Three of these strains (ST11, ST23 and ST340) belong to the clonal group (CG) 258, which has been globally described as a KPC spreader and is often related to carbapenemase production. Therefore, strains with STs grouped into this CG are classified as international high-risk clones (Munoz-Price et al. 2013; Gonçalves et al. 2017; Azevedo et al. 2019). In fact, among the eight strains harbouring $b l a_{\mathrm{KPC}}$ gene in this study, seven belong to CG258. K. pneumoniae strains belonging to CG258 harbouring virulence and resistance genes have been described causing both hospital and community infections (Azevedo et al. 2019). ST11 was found in three strains in the study (KpAm01, KpAm02 and KpAm13), all classified as XDR, this ST is widespread in Brazil and is internationally considered a high-risk clone. The ST340 was found in three strains (KpAm06, KpAm08 and KpAm09) with XDR profile in the first two and MDR in the last, and is also known to be widespread in Brazil (Gonçalves et al. 2017) (Fig. 2).

In a study of the genomic population of $K$. pneumoniae, Wyres et al. (2020) describes the six hyper-virulent global problem clones, the ST23 found in our strains belongs to this group. The KpAm11 and KpAm24 (ST23), both isolated from tracheal secretion, have the HMV phenotype harboring several virulence genes and wzi $\mathrm{K} 1$, indicating that these strains are hyper-virulent; several other studies describe ST23 worldwide as a hyper-virulent clone (Cheng et al. 2015; Mukherjee et al. 2020; Pereira et al. 2017). Moreover, the ST23 was previously described in Brazil in a colistin-resistant $K$. pneumoniae harboring $b l a_{\mathrm{KPC}}$ and several other resistance genes (Boszczowski et al. 2019), Coutinho et al. (2014) reported an invasive liver abscess syndrome caused by $K$. pneumoniae clone ST23 and also in non-human primates (Alouatta clamitans) with hypervirulent and hyper-mucoid characteristics (Anzai et al. 2017). The ST378 and ST462 are double-locus variants of the ST23 and appear in few studies, composing the bacterial collection among several STs (Lin et al. 2016; Zhong et al. 2014; Yan et al. 2015; Zhan et al. 2021; Saxenborn et al. 2021). KpAm16 presented ST307 and Wyres et al. (2019) emphasizes the need for more attentive epidemiological surveillance of this clone, due to its rapid and emerging global spread. This ST was also reported in other studies in southeastern Brazil as described by Dropa et al. (2016), that found K. pneumoniae harboring CTX-M-15 from wastewater, and Sartori et al. (2019) related MDR CTX-M-15 causing urinary tract infection in a dog. ST277 is a double-locus variant ST258, and was previously reported by Chmelnitsky et al. (2013) in two $K$. pneumoniae ST277 carrying $b l a_{\mathrm{KPC}}$ gene and the isolate KpAm03 presented the same characteristic. The ST198 and ST502 grouped into smaller CGs, the first one was found in the strains KpAm14, KpAm17 and KpAm21 and was previously described in Brazil in K. pneumoniae producing CTX-M-15 isolated from commercial lettuce (Lopes et al. 2017).

The ICE $K p$ is a mobile genetic element associated with invasive infection in $K$. pneumoniae (Lam et al. 2018), where ICE $K p 12$ with $y b t$ lineage 60 were identified in the WGS analyses in the three ST198 strains. The ST502 was found in three MDR strains (KpAm12, KpAm18 and KpAm19), this ST has been described to cause infections in humans, including community infections in Brazil (Azevedo et al. 2019). Three new STs were described in this study, ST3991 (KpAm22) due to a new allele number combination, ST3993 (KpAm10), due to the new infB (177), $m d h$ (298) and phoE (417) alleles and ST5209 (KpAm05), due the new tonB (705) allele. Through data analysis by the curators of the MLST database, the KpAm10 strain had its species re-identified as Klebsiella quasipneumoniae subsp. quasipneumoniae and was deposited in the database with this identification. Accession numbers of all WGS strains are listed in Supplementary Table 1 .

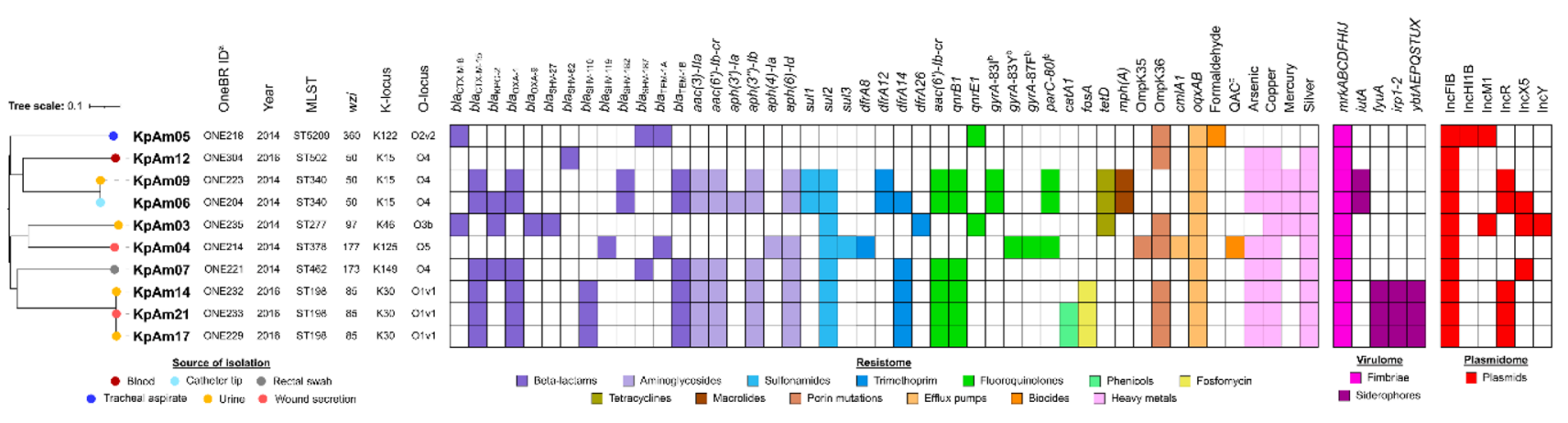

Fig. 2 Phylogenetic relations, resistome, virulome and plasmidome of 10 Klebsiella pneumoniae isolates from clinical sources in Manaus, Brazil. aneBR ID refers to the ID of the isolates in the One Health
Brazilian Resistance Integrated Genomic database (OneBR). ${ }^{b}$ Mutations in quinolone resistance determining regions. ${ }^{\mathrm{c}} Q A C$ quaternary ammonium compounds 


\section{Conclusion}

The results presented in this study showed that more than $80 \%$ of the studied strains isolated from inpatients in the city of Manaus, located in the state of Amazonas in Brazil were resistant to several antimicrobials. The majority of strains were classified as MDR and five strains as XDR. Moreover, several virulence and antimicrobial-resistance genes were found and eight strains presented the hyper-mucoviscous phenotype. MLST analysis demonstrated a great diversity among the strains, totaling 12 different STs including highrisk international clones belonging to CG258.

In 2020, Manaus suffered a collapse in the public health system due to the COVID-19 pandemic and a large number of patients from all over the region were admitted to the city hospitals undergoing massive antimicrobial therapies. Although the number of strains evaluated in this study is relatively small and they were isolated before the pandemic period, they come from different hospitals suggesting a worrying scenario. Therefore, after the COVID-19 pandemic emergency, further studies aiming to elucidate and prevent the spread of hyper-virulent and multidrug-resistant bacteria in the hospitals of this region will be extremely important.

\section{Data}

This Whole Genome Shotgun project has been deposited at DDBJ/ENA/GenBank, and genomic information of K. pneumoniae strains are available on the OneBR platform http:// onehealthbr.com/ (Table supplementary 1).

Supplementary Information The online version contains supplementary material available at https://doi.org/10.1007/s00203-022-02813-0.

Acknowledgements The authors thank the team of curators from the Institut Pasteur MLST and genome MLST databases for curating the data and making them publicly available at http://bigsdb.pasteur.fr. We thank Bill \& Melinda Gates Foundation, FAPESP, CAPES and CNPq research grants are gratefully acknowledged.

Author contributions AP-S conceived and designed the experiments. $\mathrm{RN}-\mathrm{S}$ conducted all experiments relating to antimicrobial resistance, virulence, MLST and wrote the manuscript. LC conducted the epidemiological and bioinformatics analyses. KRCC provided the bacterial isolates with their respective information. MO-S supported in the antimicrobial susceptibility experiments. BF and QM carried out the genome sequencing. ES, FE and NL performed genomic and bioinformatics analyzes especially in the analysis of resistome, virulome and plasmidome and in the deposit of genomic data. KW contributed especially in the analysis and writing of the manuscript. All authors reviewed and approved the final manuscript.

Funding This work was supported by the Fundação de Amparo à Pesquisa do Estado de São Paulo (FAPESP) [Grant No. 2013/22581-5]. RNS is a PhD fellow of CAPES, therefore, this study was financed in part by the Coordenação de Aperfeiçoamento de Pessoal de Nível Superior [Grant No 88882365161 and Finance Code 001]. BF is a research fellow of fellow of (CAPES) [Grant No. 88887.358057/201900]. FE is a research fellow of FAPESP [Grant No. 2019/15778-4]. N.L. is a research fellow of Conselho Nacional de Desenvolvimento Científico e Tecnológico (CNPq) [Grant No. 312249/2017-9].

\section{Declarations}

Conflict of interest The authors have declared that no competing interests exist.

Ethical approval Ethical approval was received from the School of Pharmaceutical Sciences of Ribeirão Preto, University of São Paulo (Ribeirão Preto, SP, Brazil) [approval no. CEP/FCFRP 362; CAEE 36031914.9.0000.5403].

\section{References}

Anzai EK, de Souza Júnior JC, Peruchi AR, Fonseca JM, Gumpl EK, Pignatari ACC, Hirano ZMB, Silveira ACO (2017) First case report of non-human primates (Alouatta clamitans) with the hypervirulent Klebsiella pneumoniae serotype K1 strain ST 23: a possible emerging wildlife pathogen. J Med Primatol 46:337-342. https://doi.org/10.1111/jmp.12296

Azevedo PAA, Furlan JPR, Gonçalves GB, Gomes CN, Goulart RDS, Stehling EG, Pitondo-Silva A (2019) Molecular characterisation of multidrug-resistant Klebsiella pneumoniae belonging to CC258 isolated from outpatients with urinary tract infection in Brazil. $\mathbf{J}$ Glob Antimicrob Resist 18:74-79. https://doi.org/10.1016/j.jgar. 2019.01.025

Bengoechea JA, Sa Pessoa J (2019) Klebsiella pneumoniae infection biology: living to counteract host defences. FEMS Microbiol Rev 43:123-144. https://doi.org/10.1093/femsre/fuy043

Bortolaia V, Kaas RS, Ruppe E, Roberts MC, Schwarz S, Cattoir V, Philippon A, Allesoe RL, Rebelo AR, Florensa AF, Fagelhauer L, Chakraborty T, Neumann B, Werner G, Bender JK, Stingl K, Nguyen M, Coppens J, Xavier BB, Malhotra-Kumar S, Westh H, Pinholt M, Anjum MF, Duggett NA, Kempf I, Nykäsenoja S, Olkkola S, Wieczorek K, Amaro A, Clemente L, Mossong J, Losch S, Ragimbeau C, Lund O, Aarestrup FM (2020) ResFinder 4.0 for predictions of phenotypes from genotypes. J Antimicrob Chemother 75:3491-3500. https://doi.org/10.1093/jac/dkaa345

Boszczowski I, Salomão MC, Moura ML, Freire MP, Guimarães T, Cury AP, Rossi F, Rizek CF, Martins RCR, Costa SF (2019) Multidrug-resistant Klebsiella pneumoniae: genetic diversity, mechanisms of resistance to polymyxins and clinical outcomes in a tertiary teaching hospital in Brazil. Rev Inst Med Trop Sao Paulo 61:e29. https://doi.org/10.1590/S1678-9946201961029

Campos TA, Gonçalves LF, Magalhães KG, de Paulo MV, Pappas Júnior GJ, Peirano G, Pitout JDD, Gonçalves GB, Furlan JPR, Stehling EG, Pitondo-Silva AA (2018) A fatal bacteremia caused by hypermucousviscous KPC-2 producing extensively drug-resistant K64-ST11 Klebsiella pneumoniae in Brazil. Front Med (lausanne) 21:265. https://doi.org/10.3389/fmed.2018.00265

Candan ED, Aksöz N (2015) Klebsiella pneumoniae: characteristics of carbapenem resistance and virulence factors. Acta Biochim Pol 62:867-874. https://doi.org/10.18388/abp.2015_1148

Carattoli A, Zankari E, García-Fernández A, Voldby Larsen M, Lund O, Villa L, Møller Aarestrup F, Hasman H (2014) In silico detection and typing of plasmids using PlasmidFinder and plasmid 
multilocus sequence typing. Antimicrob Agents Chemother 58:3895-3903. https://doi.org/10.1128/AAC.02412-14

Catalán-Nájera JC, Garza-Ramos U, Barrios-Camacho H (2017) Hypervirulence and hypermucoviscosity: two different but complementary Klebsiella spp. phenotypes? Virulence 8:1111-1123. https://doi.org/10.1080/21505594.2017.1317412

Cheng KC, Lee MF, Chuang YC, Yu WL (2015) First description of lung abscess caused by ST23 clone capsule genotype K1 Klebsiella pneumoniae. J Formos Med Assoc 114:379-380. https:// doi.org/10.1016/j.jfma.2013.08.008

Chmelnitsky I, Shklyar M, Hermesh O, Navon-Venezia S, Edgar $\mathrm{R}$, Carmeli Y (2013) Unique genes identified in the epidemic extremely drug-resistant KPC-producing Klebsiella pneumoniae sequence type 258. J Antimicrob Chemother 68:74-83. https:// doi.org/10.1093/jac/dks370.Erratum.In:JAntimicrobChemother. 68,2178

Clinical and Laboratory Standards Institute (2020) Performance standards for antimicrobial susceptibility testing, M100. 30th ed

Coutinho RL, Visconde MF, Descio FJ, Nicoletti AG, Pinto FC, Silva AC, Rodrigues-Costa F, Gales AC, Furtado GH (2014) Community-acquired invasive liver abscess syndrome caused by a K1 serotype Klebsiella pneumoniae isolate in Brazil: a case report of hypervirulent ST23. Mem Inst Oswaldo Cruz 109:970-971. https://doi.org/10.1590/0074-0276140196

Dropa M, Lincopan N, Balsalobre LC, Oliveira DE, Moura RA, Fernandes MR, da Silva QM, Matté GR, Sato MI, Matté MH (2016) Genetic background of novel sequence types of CTX-M-8- and CTX-M-15-producing Escherichia coli and Klebsiella pneumoniae from public wastewater treatment plants in São Paulo Brazil. Environ Sci Pollut Res Int 23:4953-4958. https://doi.org/10.1007/ s11356-016-6079-5

Ferrante L, Fearnside PM (2020) Protect indigenous peoples from COVID-19. Science 368:251. https://doi.org/10.1126/science. abc0073

Ferreira RL, da Silva BCM, Rezende GS, Nakamura-Silva R, PitondoSilva A, Campanini EB, Brito MCA, da Silva EML, Freire CCM, da Cunha AF, Pranchevicius MDS (2019) High prevalence of multidrug-resistant Klebsiella pneumoniae harboring several virulence and $\beta$-lactamase encoding genes in a Brazilian Intensive Care Unit. Front Microbiol 9:3198. https://doi.org/10.3389/ fmicb.2018.03198

Garza-Ramos U, Silva-Sanchez J, Barrios H, Rodriguez-Medina N, Martínez-Barnetche J, Andrade V (2015) Draft genome sequence of the first hypermucoviscous Klebsiella variicola clinical isolate. Genome Announc 3:e01352-e1414. https://doi.org/10.1128/ genomeA.01352-14

Gonçalves GB, Furlan JPR, Vespero EC, Pelisson M, Stehling EG, Pitondo-Silva A (2017) Spread of multidrug-resistant high-risk Klebsiella pneumoniae clones in a tertiary hospital from southern Brazil. Infect Genet Evol 56:1-7. https://doi.org/10.1016/j. meegid.2017.10.011

IBGE. Brazilian National Institute of Geography and Statistics (2019) Gross Domestic Product-2017. https://cidades.ibge.gov.br/brasil/ am/manaus/panorama. Accessed 13 Dec 2019

Kaas RS, Leekitcharoenphon P, Aarestrup FM, Lund O (2014) Solving the problem of comparing whole bacterial genomes across different sequencing platforms. PLoS ONE 9:e104984. https://doi.org/ 10.1371/journal.pone.0104984

Kuş H, Arslan U, Türk Dağ 1 H, Findık D (2017) Hastane enfeksiyonu etkeni Klebsiella pneumoniae izolatlarında çeşitli virülans faktörlerinin araştırılması [Investigation of various virulence factors of Klebsiella pneumoniae strains isolated from nosocomial infections]. Mikrobiyol Bul 51:329-339. https://doi.org/10.5578/ mb.59716

Lam MMC, Wick RR, Wyres KL, Gorrie CL, Judd LM, Jenney AWJ, Brisse S, Holt KE (2018) Genetic diversity, mobilisation and spread of the yersiniabactin-encoding mobile element ICEKp in Klebsiella pneumoniae populations. Microb Genom 4:e00196. https://doi.org/10.1099/mgen.0.000196

Lam MMC, Wick RR, Watts SC, Cerdeira LT, Wyres KL, Holt KE (2021) A genomic surveillance framework and genotyping tool for Klebsiella pneumoniae and its related species complex. Nat Commun 12:4188. https://doi.org/10.1038/s41467-021-24448-3

Lee CH, Liu JW, Su LH, Chien CC, Li CC, Yang KD (2010) Hypermucoviscosity associated with Klebsiella pneumoniae-mediated invasive syndrome: a prospective cross-sectional study in Taiwan. Int J Infect Dis 14:e688-e692. https://doi.org/10.1016/j.ijid.2010. 01.007

Letunic I, Bork P (2021) Interactive Tree Of Life (iTOL) v5: an online tool for phylogenetic tree display and annotation. Nucleic Acids Res 49(W1):W293-W296. https://doi.org/10.1093/nar/gkab301

Lin WP, Wang JT, Chang SC, Chang FY, Fung CP, Chuang YC, Chen YS, Shiau YR, Tan MC, Wang HY, Lai JF, Huang IW, Lauderdale TL (2016) The antimicrobial susceptibility of Klebsiella pneumoniae from community settings in Taiwan, a trend analysis. Sci Rep 6:36280. https://doi.org/10.1038/srep36280

Lopes R, Cerdeira LT, Fernandes MR, Pérez-Chaparro PJ, McCulloch JA, Lincopan N (2017) Draft genome sequence of a CTX-M15-producing endophytic Klebsiella pneumoniae ST198 isolate from commercial lettuce. J Glob Antimicrob Resist 10:19-20. https://doi.org/10.1016/j.jgar.2017.03.005

Magiorakos AP, Srinivasan A, Carey RB, Carmeli Y, Falagas ME, Giske CG, Harbarth S, Hindler JF, Kahlmeter G, Olsson-Liljequist B, Paterson DL, Rice LB, Stelling J, Struelens MJ, Vatopoulos A, Weber JT, Monnet DL (2012) Multidrug-resistant, extensively drug-resistant and pandrug-resistant bacteria: an international expert proposal for interim standard definitions for acquired resistance. Clin Microbiol Infect 18:268-281. https://doi.org/10.1111/j. 1469-0691.2011.03570.x

Mathers AJ, Peirano G, Pitout JD (2015) The role of epidemic resistance plasmids and international high-risk clones in the spread of multidrug-resistant Enterobacteriaceae. Clin Microbiol Rev 28:565-591. https://doi.org/10.1128/CMR.00116-14

Mohammed ES, Flayyih MT (2018) Detection of rmpA and magA genes and hypermucoviscosity phenotype in Klebsiella pneumoniae isolated from water samples in compare with clinical isolates. Curr Res Microbiol Biotechnol 6:1424-1430

Mukherjee S, Naha S, Bhadury P, Saha B, Dutta M, Dutta S, Basu S (2020) Emergence of OXA-232-producing hypervirulent Klebsiella pneumoniae ST23 causing neonatal sepsis. J Antimicrob Chemother 75:2004-2006. https://doi.org/10.1093/jac/dkaa080

Munoz-Price LS, Poirel L, Bonomo RA, Schwaber MJ, Daikos GL, Cormican M, Cornaglia G, Garau J, Gniadkowski M, Hayden MK, Kumarasamy K, Livermore DM, Maya JJ, Nordmann P, Patel JB, Paterson DL, Pitout J, Villegas MV, Wang H, Woodford N, Quinn JP (2013) Clinical epidemiology of the global expansion of Klebsiella pneumoniae carbapenemases. Lancet Infect Dis 13:785-796. https://doi.org/10.1016/S1473-3099(13)70190-7

Nakamura-Silva R, Domingues Macedo LM, Cerdeira LT, OliveiraSilva M, Silva-Sousa YTC, Pitondo-Silva A (2021a) First report of hypermucoviscous Klebsiella variicola subsp. variicola causing primary endodontic infection. Clin Microbiol Infect 27:303-304. https://doi.org/10.1016/j.cmi.2020.07.045

Nakamura-Silva R, Oliveira-Silva M, Furlan JPR, Stehling EG, Miranda CES, Pitondo-Silva A (2021b) Characterization of multidrug-resistant and virulent Klebsiella pneumoniae strains belonging to the high-risk clonal group 258 (CG258) isolated from inpatients in northeastern Brazil. Arch Microbiol 203(7):4351-4359. https://doi.org/10.1007/s00203-021-02425-0

Pereira A, Petrucci T, Simões MJ (2017) Klebsiella pneumoniae do Serotipo K1 e do clone Hipervirulento ST23: primeiro caso documentado em Portugal [Klebsiella pneumoniae from K1 and 
hypervirulent clone ST23: first documented case in Portugal]. Acta Med Port 30:496-499. https://doi.org/10.20344/amp.7705

Pereira JL, Volcão LM, Klafke GB, Vieira RS, Gonçalves CV, Ramis IB, da Silva PEA, von Groll A (2019) Antimicrobial resistance and molecular characterization of extended-spectrum $\beta$-lactamases of Escherichia coli and Klebsiella spp. isolates from urinary tract infections in Southern Brazil. Microb Drug Resist 25:173-181. https://doi.org/10.1089/mdr.2018.0046

Remya PA, Shanthi M, Sekar U (2019) Characterisation of virulence genes associated with pathogenicity in Klebsiella pneumoniae. Indian J Med Microbiol 37:210-218. https://doi.org/10.4103/ ijmm.IJMM_19_157

Ribas RM, Campos PA, Brito CS, Gontijo-Filho PP (2020) Coronavirus Disease 2019 (COVID-19) and healthcare-associated infections: emerging and future challenges for public health in Brazil. Travel Med Infect Dis 37:101675. https://doi.org/10.1016/j.tmaid. 2020.101675

Sartori L, Sellera FP, Moura Q, Cardoso B, Cerdeira L, Lincopan N (2019) Multidrug-resistant CTX-M-15-positive Klebsiella pneumoniae ST307 causing urinary tract infection in a dog in Brazil. J Glob Antimicrob Resist 19:96-97. https://doi.org/10.1016/j.jgar. 2019.09.003

Saxenborn P, Baxter J, Tilevik A, Fagerlind M, Dyrkell F, Pernestig AK, Enroth H, Tilevik D (2021) Genotypic characterization of clinical Klebsiella spp isolates collected from patients with suspected community-onset sepsis Sweden. Front Microbiol 12:640408. https://doi.org/10.3389/fmicb.2021.640408

Walker KA, Miller VL (2020) The intersection of capsule gene expression, hypermucoviscosity and hypervirulence in Klebsiella pneumoniae. Curr Opin Microbiol 54:95-102. https://doi.org/10. 1016/j.mib.2020.01.006

Weisburg WG, Barns SM, Pelletier DA, Lane DJ (1991) 16S ribosomal DNA amplification for phylogenetic study. J Bacteriol 173:697703. https://doi.org/10.1128/jb.173.2.697-703.1991

Wick RR, Judd LM, Gorrie CL, Holt KE (2017) Unicycler: resolving bacterial genome assemblies from short and long sequencing reads. PLoS Comput Biol 13:e1005595. https://doi.org/10.1371/ journal.pcbi.1005595

Wiskur BJ, Hunt JJ, Callegan MC (2008) Hypermucoviscosity as a virulence factor in experimental Klebsiella pneumoniae endophthalmitis. Invest Ophthalmol vis Sci 49:4931-4938. https:// doi.org/10.1167/iovs.08-2276

Wyres K, Holt KE (2018) Klebsiella pneumoniae as a key trafficker of drug resistance genes from environmental to clinically important bacteria. Curr Opin Microbiol 45:131-139. https://doi.org/10. 1016/j.mib.2018.04.004

Wyres KL, Hawkey J, Hetland MAK, Fostervold A, Wick RR, Judd LM, Hamidian M, Howden BP, Löhr IH, Holt KE (2019) Emergence and rapid global dissemination of CTX-M-15-associated Klebsiella pneumoniae strain ST307. J Antimicrob Chemother 74(3):577-581. https://doi.org/10.1093/jac/dky492

Wyres KL, Lam MMC, Holt KE (2020) Population genomics of Klebsiella pneumoniae. Nat Rev Microbiol 18(6):344-359. https://doi. org/10.1038/s41579-019-0315-1

Yan JJ, Wang MC, Zheng PX, Tsai LH, Wu JJ (2015) Associations of the major international high-risk resistant clones and virulent clones with specific ompK36 allele groups in Klebsiella pneumoniae in Taiwan. New Microbes New Infect 5:1-4. https://doi.org/ 10.1016/j.nmni.2015.01.002

Yu WL, Ko WC, Cheng KC, Lee HC, Ke DS, Lee CC, Fung CP, Chuang YC (2006) Association between rmpA and magA genes and clinical syndromes caused by Klebsiella pneumoniae in Taiwan. Clin Infect Dis 42(10):1351-1358. https://doi.org/10.1086/ 503420

Zhan Q, Xu Y, Wang B, Yu J, Shen X, Liu L, Cao X, Guo Y, Yu F (2021) Distribution of fluoroquinolone resistance determinants in Carbapenem-resistant Klebsiella pneumoniae clinical isolates associated with bloodstream infections in China. BMC Microbiol 21:164. https://doi.org/10.1186/s12866-021-02238-7

Zhong X, Xu H, Chen D, Zhou H, Hu X, Cheng G (2014) First emergence of acr $\mathrm{AB}$ and oqx $\mathrm{AB}$ mediated tigecycline resistance in clinical isolates of Klebsiella pneumoniae pre-dating the use of tigecycline in a Chinese hospital. PLoS ONE 9:e115185. https:// doi.org/10.1371/journal.pone.0115185

Publisher's Note Springer Nature remains neutral with regard to jurisdictional claims in published maps and institutional affiliations. 